\title{
Investigation of Induced Unbalance Magnitude on Dynamic Characteristics of High-speed Turbocharger with Floating Ring Bearings
}

\author{
Guang-Fu Bin ${ }^{1}$, Yuan Huang ${ }^{1}$, Shuai-Ping Guo ${ }^{1}$ Xue-Jun Li ${ }^{* *}$ and Gang Wang ${ }^{2}$
}

\begin{abstract}
Due to operational wear and uneven carbon absorption in compressor and turbine wheels, the unbalance (me) vibration is induced and could lead to sub-synchronous vibration accidents for high-speed turbocharger (TC). There are very few research works that focus on the magnitude effects on such induced unbalance vibration. In this paper, a finite element model (FEM) is developed to characterize a realistic automotive TC rotor with floating ring bearings (FRBs). The nonlinear dynamic responses of the TC rotor system with different levels of induced unbalance magnitude in compressor and turbine wheels are calculated. From the results of waterfall and response spectral intensity plots, the bifurcation and instability phenomena depend on unbalance magnitude during the startup of TC. The sub-synchronous component $0.12 \times$ caused rotor unstable is the dominant frequency for small induced unbalance. The nonlinear effects of induced unbalance in the turbine wheel is obvious stronger than the compressor wheel. As the unbalance magnitude increases from $0.05 \mathrm{~g} \cdot \mathrm{mm}$ to $0.2 \mathrm{~g} \cdot \mathrm{mm}$, the vibration component changes from mainly $0.12 \times$ to synchronous vibration $1 \times$. When unbalance increases continuously, the rotor vibration response amplitude is rapidly growing and the $1 \times$ caused by the large unbalance excitation becomes the dominant frequency. A suitable un-balance magnitude of turbine wheel and compressor wheel for the high-speed TC rotor with FRBs is proposed: the value of induced un-balance magnitude should be kept around $0.2 \mathrm{~g} \cdot \mathrm{mm}$.
\end{abstract}

Keywords: High-speed TC with FRBs, Induced unbalance magnitude, Nonlinear dynamic response, Sub-synchronous vibration, Stability

\section{Introduction}

High-speed turbochargers (TCs) have gained significant attention in recent years. They already have been widely used in commercial vehicles. The weight of a typical automotive $\mathrm{TC}$ is about $1 \mathrm{~kg}$. It has $200 \mathrm{~mm}$ in length and 5-15 $\mathrm{mm}$ in diameter and operates above $100000 \mathrm{r} / \mathrm{min}$, which fails in the high-speed and lightweight rotor category. A small TC rotor with small unbalance can produce large vibration when it spins at

\footnotetext{
*Correspondence: abin811025@163.com

${ }^{1}$ Health Maintenance for Mechanical Equipment Key Lab of Hunan Province, Hunan University of Science and Technology, Xiangtan 411201, China

Full list of author information is available at the end of the article
}

high speed [1]. It is well known that the first exhaust TC was invented at the beginning of last century. There are many efforts to investigate the rotordynamics of TC rotor-bearing system $[2,3]$. The floating ring bearing (FRB) configuration is the simplest bearing design and the most widely used in small TCs. In such FRB design, two fluid films are connected in series in a floating ring, which are capable of providing higher damping and fewer friction losses than a single-film plain journal bearing [4]. However, due to its high speed and high nonlinear feature, the FRB system exhibits high magnitude sub-synchronous vibration for a wide speed range [5]. The bearing fluid-film whirl instability is the main source of the sub-synchronous vibration. The nonlinear reaction forces inside the bearings are usually causing 
the $\mathrm{TC}$ rotor to whirl in a limit cycle but may become large enough to cause permanent damages. Based on classical linear eigenvalue analysis presented by Kirk et al. [6], both inner and outer films always lead to the instability for high-speed TCs. Li et al. [7] developed a stability analysis and an identification method for rotorbearings system. Surprisingly, because of high nonlinear characteristics of the inner and outer oil films, it was observed that the loss of instability can induce the onset of stable limit cycles under sub-synchronous frequency via a Hopf bifurcation [8], which can always ensure the safe operation of a TC [9]. Interestingly, according to the published simulation and experimental results $[10,11]$, it has been shown that the proved the final occurrence of the above-mentioned possibilities is sensitive to the rotor-FRB system physical parameters of TC, such as bearing structural parameters, oil feeding conditions and imposed unbalance values. The deep sensitivity clearly implies the possibility to optimize the $\mathrm{TC}$ rotor dynamic response and reduce the vibration by adjusting the physical structural parameters of a TC.

Rotor unbalance is a classic structural parameter which can induce the vibration for the high-speed TC rotor systems. Zhai et al. [12] analyzed the shaft dynamic response due to the unbalanced mass using the finite element analysis. Gunter et al. [13] studied the effects of rotor unbalance in the compressor and turbine wheels. These effects can strongly influence the limit cycle orbits. Alsaeed [14], presented a method to suppress the sub-synchronous vibrations by inducing the TC rotor unbalance. Sterling et al. [15] developed a theory to study the effect of induced unbalance on sub-synchronous vibration of an automotive TC. It was shown that an increasing unbalance can cause a reduction in the sub-synchronous vibration. Kirk et al. [16] also investigated the influence of TC unbalance on subsynchronous vibration amplitude. Tian et al. [17] developed the influence of unbalance on the rotordynamic characteristics of a real TC-FRB system over the speed range from $0 \mathrm{~Hz}$ to $3500 \mathrm{~Hz}$, and confirmed the unbalance magnitude is a critical parameter for the system response. Yao et al. [18] developed a method to suppress the multi-frequency rotor vibration using electromagnetic force. Other methods are also developed for rotor machine $[19,20]$. In a word, many researchers devoted to investigate the influence of the unbalance level on TC dynamics at a fixed unbalance location. Appropriate unbalance can suppress the sub-synchronous vibration and improve the response behavior of $\mathrm{TC}$ as many researchers mentioned, which is determined by the special structure, high speed operation conditions, and nonlinear FRB. However, the nonlinear analysis is also rare at high speed for the small-sized and light-weight TC rotor with FRB. The influence of unbalance magnitude on the rotordynamic characteristics has not performed adequately.

Interestingly, the turbine impeller may exist some uncertain coke deposition in the extreme running condition with high temperature and variational operation speed mode. Thus, the induced unbalance in the turbine and compressor impellers can be formed after running a certain period for the high-speed TC. Furthermore, the unbalance can be grown with the running time, which directly influences the high-efficiency operation of highspeed TC and even causes serious nonlinear vibration accident. Therefore, it is necessary to investigate the effects of induced unbalance magnitude on the dynamic characteristics and reduce the vibration to an acceptable level by controlling the unbalance magnitude for the high-speed TC with FRB.

Major steps are outlined below. First, a FEM is required to characterize the TC rotor dynamics. The logarithmic decrements and modes are used to determine the TC rotor system stability. Second, based on the frequency domain of time transient analysis from the FEM, the nonlinear dynamic responses of the $\mathrm{TC}$ rotor system with different levels of induced unbalance magnitude in compressor and turbine wheels are calculated. Finally, the waterfall and response spectral intensity plots are compared to propose a suitable unbalance magnitude of turbine wheel and compressor wheel for the high-speed TC rotor with FRBs. In summary, the unbalance magnitude is an effective way to achieve the small vibration and stable operation for the high-speed TC rotor with FRBs.

The article proceeds as follows. First, the TC rotor and bearing model is described. Then, a realistic automotive TC rotor with FRB is taken as an example and its dynamic finite element model is presented to characterize the dynamic of rotor. Moreover, the rotor stability analysis is discussed. This is followed by simulation and discussion. Conclusions are given in the last section.

\section{TC Rotor and Bearing Model Description}

The TC rotor usually consists of a radial outflow compressor wheel and a radial inflow turbine wheel on a single shaft. Bearings are mounted inboard, with the compressor and turbine overhung, as shown in Figure 1. FRB consists of two hydrodynamic fluid film bearings in series with the ring as an additional degrees-of-freedom (DOF). The inner film has two rotating surfaces, i.e., journal and ring with rotational speeds of $\Omega_{j}$ and $\Omega_{r}$, and the outer film has only one rotating surface, i.e., ring with rotational speed of $\Omega_{r}$. This arrangement can only be used for high speed and light weight applications, such as in the automotive industry. 


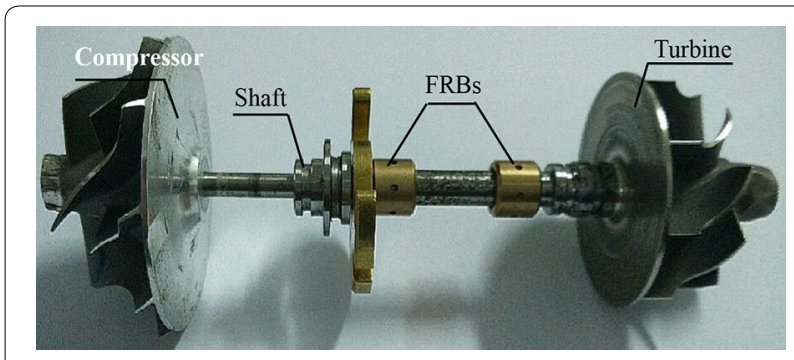

Figure 1 Automotive TC rotor supported with FRBs

\subsection{FRB Model}

Unlike conventional plain journal bearing, the floating ring rotates with the sum of speed of inner and outer hydrodynamic oil films, which is a key parameter to the analysis of TC rotor's dynamic characteristics. However, the rotational speed of floating ring is very difficult to monitor in the actual working condition. For the purpose of simplicity, the bearing feeding holes are not included and the oil feeding conditions are not considered in the FRB model. In addition, the isothermal fluid flow condition is assumed for the proposed model. The Reynolds equations for the inner and outer oil films can be written as follows:

$$
\begin{aligned}
\frac{1}{R_{j}^{2}} & \frac{\partial}{\partial \theta_{i}}\left(\frac{h_{i}^{3}}{12 \mu_{i}} \frac{\partial p_{i}}{\partial \theta_{i}}\right)+\frac{\partial}{\partial Z_{i}}\left(\frac{h_{i}^{3}}{12 \mu_{i}} \frac{\partial p_{i}}{\partial Z_{i}}\right) \\
= & \frac{\Omega_{j}+\Omega_{r}}{2} \frac{\partial h_{i}}{\partial \theta_{i}}+\frac{\partial h_{i}}{\partial t}, \\
\frac{1}{R_{o}^{2}} & \frac{\partial}{\partial \theta_{o}}\left(\frac{h_{o}^{3}}{12 \mu_{o}} \frac{\partial p_{o}}{\partial \theta_{o}}\right)+\frac{\partial}{\partial Z_{o}}\left(\frac{h_{o}^{3}}{12 \mu_{o}} \frac{\partial p_{o}}{\partial Z_{o}}\right) \\
& =\frac{\Omega_{r}}{2}+\frac{\partial h_{o}}{\partial \theta_{o}}+\frac{\partial h_{o}}{\partial t},
\end{aligned}
$$

where subscripts $i$ and $o$ identify the parameters of the inner oil film and outer oil film, respectively. Subscripts $j$ and $r$ distinguish the parameters between journal and floating rings. $p$ is the oil film pressure, and $\mu$ denotes the lubricating oil film viscosity. $R_{j}$ and $R_{o}$ correspond to the radius of journal and outer floating ring, respectively. $\theta$ is the angular coordinate for the inner and outer oil films. $Z_{i}$ and $Z_{o}$ denote the axial coordinates of the inner and outer films, respectively. $h_{i}$ and $h_{o}$ represent the oil film thicknesses of inner and outer, respectively. $\Omega_{j}$ and $\Omega_{r}$ are the angular velocity of journal and floating ring, respectively.

The equilibrium positions are determined by an iterative procedure, the linearized bearing coefficients of inner film and outer film can be obtained from Eqs. (1) and (2). Actually, the FRB is usually used for very high-speed applications and the floating ring is designed to have high ring speed to ensure the proper hydrodynamic lubrication. At very high speed, the eccentricity ratios are very small and the journal, ring, and bearing are nearly concentric. When the eccentricity ratios are zero, the journal torque $T_{j}$ and ring torque $T_{r}$ balance becomes

$$
T_{j}=2 \pi \mu_{i} R_{i}^{3}\left(\Omega_{i}-\Omega_{o}\right) L_{i} / C_{i}=T_{r}=2 \pi \mu_{o} R_{o}^{3} \Omega_{o} L_{o} / C_{o} .
$$

Thus, the ring speed $\Omega_{r}$ can be determined from the above Eq. (3). Once the ring speed $\Omega_{r}$ is known, the two Reynolds equations for the inner and outer films can be solved separately and the bearing coefficients can be determined just like the conventional plain cylindrical bearing for linear analysis. The maximum possible ring speed $\Omega_{r}$ can be conveniently estimated using the torque balance with concentric journal, ring, and bearing [21]:

$$
\Omega_{r}=\frac{\Omega_{j}}{1+\left[\left(\mu_{o} / \mu_{i}\right)\left(R_{o} / R_{i}\right)^{3}\left(L_{o} / L_{i}\right)\left(C_{i} / C_{o}\right)\right]},
$$

where $\mu$ is the oil dynamic viscosity, $L$ is the axial length of the bearing, and $C$ is the bearing radial clearance. Subscripts $i$ and $o$ are for the inner and outer film properties. Since the inner film has much higher surface velocity and smaller clearance, the lubricant operating temperature is higher than that of the outer film. Typical ratio of $\mu_{o} / \mu_{i}$ ranges from 1.2 to 2.0 . The clearance ratio $C_{o} / C_{i}$ is about 1.5 to 4.0 . For FRB, the clearance ratio is probably the most important parameter, the optimal value depends on the operating conditions and ratios of $R_{o} / R_{i}$ and $L_{o} / L_{i}$. Smaller clearance ratio will decrease the ring speed which is not desirable from the lubrication point of view, although it may increase the rotor stability. Large clearance ratio increases the ring speed, however, it is not desirable from rotor vibration and stability point of view. However, the nonlinear fluid film forces for the inner and outer films are dependent on the motions of the journal and ring.

\subsection{TC Rotor with Induced Unbalance}

Considering a high-speed TC rotor, since the partial differential equations given by modeling of the rotor shaft in a continuous system are difficult to be tackled, discretizing the continuum to a discretized system by the FE method. The motion governing equation for the investigated $\mathrm{TC}$ rotor-FRB system is derived as follows:

$$
\boldsymbol{M} \ddot{\boldsymbol{x}}+C_{S G} \dot{\boldsymbol{x}}+\boldsymbol{K} \boldsymbol{x}=\boldsymbol{F}(t),
$$

where $M$ represents the mass matrix contained the mass and inertia moments of the rotor with $n$ DOFs. $C_{S G}$ is the 
damping coefficients and gyroscopic matrix. $K$ is the system stiffness coefficients matrix included diagonal and cross coupled stiffness. $\boldsymbol{x}$ is the response vector included two translational and two rotational displacements at each station in the horizontal direction $X$ and the vertical direction $Y . \boldsymbol{F}(t)$ consists of unbalance force $\boldsymbol{F}_{u b}(\Omega, t)$, static gravity force $F_{s}$ in $Y$ direction and nonlinear bearing force $\boldsymbol{F}_{i}(x, \dot{x}, t)$. Thus, the $\boldsymbol{F}(t)$ can be rewritten as

$$
\boldsymbol{F}(t)=\boldsymbol{F}_{i}(x, \dot{x}, t)+\boldsymbol{F}_{u b}(\Omega, t)+\boldsymbol{F}_{s} .
$$

Considering the practical structure and the locations probability of unbalance on gasoline engine TC, the $\boldsymbol{F}_{u b}$ $(\Omega, t)$ usually can be decomposed $\boldsymbol{F}_{u b}^{c}$ and $\boldsymbol{F}_{u b}^{t}$, which exists at the end of compressor and turbine impellers, as shown in Eqs. (7) and (8). $m_{c}$ and $m_{t}$ denote the mass of the compressor and turbine impellers, respectively. $e$ is the unbalance displacement. $\phi$ is the rotor rotating angle around the $Z$ axis, and therefore, $\dot{\phi}$ and $\ddot{\phi}$ represent the angular speed and acceleration, respectively.

$$
\begin{aligned}
& \boldsymbol{F}_{u b}^{c}=\left(\begin{array}{c}
F_{u b}^{x c} \\
F_{u b}^{y c}
\end{array}\right)=\left(\begin{array}{l}
m_{c} e \dot{\phi}^{2} \cos \phi+m_{c} e \ddot{\phi} \sin \phi \\
m_{c} e \dot{\phi}^{2} \sin \phi-m_{c} e \ddot{\phi} \cos \phi
\end{array}\right), \\
& \boldsymbol{F}_{u b}^{t}=\left(\begin{array}{c}
F_{u b}^{x t} \\
F_{u b}^{y t}
\end{array}\right)=\left(\begin{array}{l}
m_{t} e \dot{\phi}^{2} \cos \phi+m_{t} e \ddot{\phi} \sin \phi \\
m_{t} e \dot{\phi}^{2} \sin \phi-m_{t} e \ddot{\phi} \cos \phi
\end{array}\right) .
\end{aligned}
$$

The residual mass unbalance of a rotating assembly is usually determined by using the multi-plane balancing machines. However, with the development of dynamic balancing technology, the balancing precision is very high, it is urgent to know how to control the unbalance magnitude for small vibration and stable operation in the design process. It is inevitably that the unbalance existed in the rotation, especially for the high-speed rotating machine. These mass unbalance locates at different locations of two impellers with variable magnitude of $m e$. As expressed in unbalance force Eqs. (7), (8) and the governing Eq. (6), it is obvious seen that the unbalance $\boldsymbol{F}_{u b}$ directly affects the motion of TC rotor and bearing system. The rotor motion may change with the variable magnitude of unbalance in turbine or compressor wheel. Thus, it is necessary to investigate the relationship between unbalance magnitude and dynamic characteristics for the small-sized and high-speed TC rotor with FRB.

\section{Dynamic Modeling for TC Rotor with FRB \\ 3.1 Dynamic Model of FRB}

The FRB is the simplest bearing and the most widely used in small TC rotor. It is basically a floating ring inserted
Table 1 Structural and lubricating parameters of FRB

\begin{tabular}{lll}
\hline No. & Parameter & Value \\
\hline 1 & Ring mass $m_{r}(\mathrm{~g})$ & 2.160 \\
2 & Inner length $L_{i}(\mathrm{~mm})$ & 3.600 \\
3 & Outer length $L_{o}(\mathrm{~mm})$ & 6.150 \\
4 & Inner diameter $D_{i}(\mathrm{~mm})$ & 6.016 \\
5 & Outer diameter $D_{0}(\mathrm{~mm})$ & 9.540 \\
6 & Shaft diameter $D_{s}(\mathrm{~mm})$ & 6.000 \\
7 & Bearing diameter $D_{b}(\mathrm{~mm})$ & 9.600 \\
8 & Inner viscosity $\eta_{i}(\mathrm{cp})$ & 7.953 \\
9 & Outer viscosity $\eta_{0}(\mathrm{cp})$ & 9.352 \\
10 & Speed ratio $\lambda$ & 0.240 \\
\hline
\end{tabular}

in the clearance space between the rotating journal and stationary bearing housing. Although the behavior of the FRB is highly nonlinear, it is desirable to determine the linearized bearing coefficients in order to perform eigenvalue analysis of TC rotor. The FRB can be linearized about the static equilibrium position, and Reynolds equations are numerically solved for linear stiffness and damping coefficients. The FRB is usually considered as two bearings in series, and two equations for each bearing are solved. The parameters and lubricating parameters of FRB are shown in Table 1. And the dynamic model of FRB is built as shown in Figure 2(a).

The two rings of FRB were modeled as rigid body, the inner and outer oil film viscosities were assumed to be constant values with the typical $15 \mathrm{~W}-40$ supply lubricant, and the ring speed ratio was taken to be 0.24 according to Eq. (4).

The result of oil film pressure profile for the turbine end bearing at speed of $160000 \mathrm{r} / \mathrm{min}$ is presented in Figure 2(b). The inner and outer surfaces of the bearing develop a very different pressure profile. The stiffness and damping coefficients for the turbine end bearings at $160000 \mathrm{r} / \mathrm{min}$ are shown in Table 2 and Table 3, respectively. It is seen that the inner oil film stiffness is an order of magnitude higher than the stiffness coefficients generated by the outer film. Note also that the inner ring
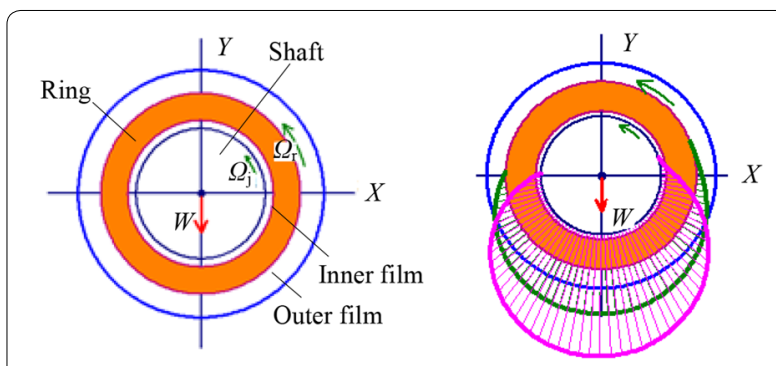

(a) FRB dynamic model

(b) Oil pressure at $160000 \mathrm{r} / \mathrm{min}$ Figure 2 Dynamic model of FRB and its oil pressure profile 
Table 2 Bearing stiffness coefficients

\begin{tabular}{lllll}
\hline Items & $\boldsymbol{K} \boldsymbol{x} \boldsymbol{x}$ & $\boldsymbol{K} \boldsymbol{x y}$ & $\boldsymbol{K y \boldsymbol { x }}$ & $\boldsymbol{K y \boldsymbol { y }}$ \\
\hline Inner film $K_{i}(\mathrm{~N} / \mathrm{mm})$ & $8.54 \times 10^{2}$ & $1.57 \times 10^{4}$ & $-2.01 \times 10^{4}$ & $4.47 \times 10^{2}$ \\
Outer film $K_{0}(\mathrm{~N} / \mathrm{mm})$ & $1.07 \times 10^{2}$ & $1.04 \times 10^{3}$ & $-1.36 \times 10^{3}$ & $1.53 \times 10^{2}$ \\
\hline
\end{tabular}

bearing cross coupling stiffness coefficients are an order of magnitude greater than the direct bearing stiffness coefficients. Obviously, the high cross coupling stiffness coefficients are the main cause of self-excited whirl motion.

Note that the FRB used for time transient numerical analysis is nonlinear, and the rotor system is also highly nonlinear. Thus, it is a very straightforward procedure for the nonlinear time transient analysis by coupling the rotor governing Eq. (5) with bearing Reynolds Eqs. (1) and (2).

\subsection{FE Modeling for TC Rotor with FRB}

The TC is a typical double overhung rotor with a steel turbine and an attached aluminum compressor impeller. That is, the turbine and compressor impellers are outboard of two bearings. The rotor shaft, FRB, shaft seal, thrust collar, shaft nut and other parts are included in the TC. All rotor components should be taken into account in the rotordynamic computation to investigate the rotor vibration response, such as the stability analysis and the frequency components in the waterfall plot. The compressor and turbine impellers are generally regarded as rigid disk to model. Due to its complex structure, the mass of rotating components, moment of inertia, and the center of gravity position can be obtained by using CAE 3-dimensional software to model and calculate, and then add to the rotor-shaft regarded as a rigid disk.

With the requirements of dynamic characteristic research and Saint-Venant principle, the FEM of rotor made reasonable simplification and assumptions, for example the turbine end component of irregular surface was defined as a cylindrical surface [22]. The FEM of a high-speed TC rotor was built according to the structural parameters and lubricating conditions, as shown in Figure 3. The TC rotor is modeled with 20 shaft finite elements which includes 42 sub-elements. Stns 9 and 12 represent the binding sites of the rotor and FRBs, respectively. Stns 21 and 22 represent the floating rings as the lumped mass $2.16 \mathrm{~g}$ of single degree of freedom. Stns 5

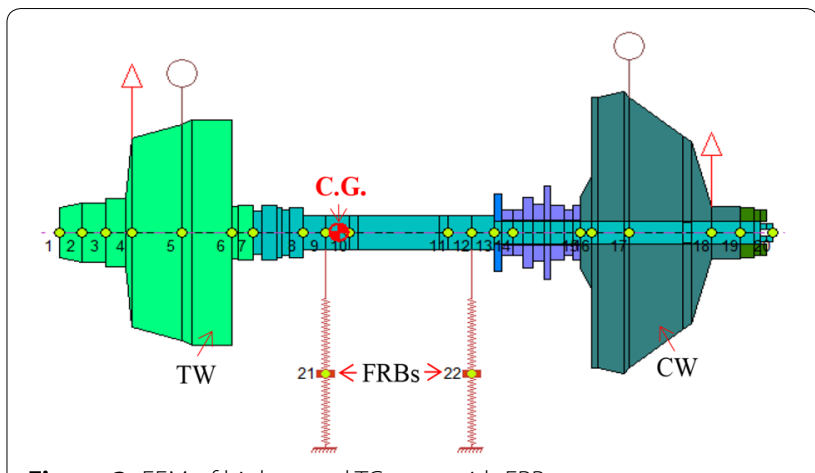

Figure 3 FEM of high-speed TC rotor with FRBs

and 17 represent the center of gravity of the compressor and turbine impellers, respectively. The turbine and compressor impellers are simplified to the tapered structure element without mass, which the mass and inertia moments are applied to the stns 5 and 17. The arrow at stn 4 represents the unbalance applied to the nose wall of compressor impeller. The circular dot near stn 9 represents the gravity center (C.G.) of the entire system which is closer to the turbine end bearing. Thus, the load of turbine end bearing is larger than the compressor end bearing.

The bearing modeling methods are different for the analysis of the various applications. For linear analysis, the bearing stiffness and damping coefficients are linearized and import by selecting the appropriate boundary conditions as mentioned before and the two rings of FRBs can be modeled as rigid body. However, for time transient numerical analysis, the vibration response can be got to solve directly the motion governing equation of $\mathrm{TC}$ rotor combined with the Reynolds equation including the bearing parameters and lubricating conditions.

\subsection{Stability Analysis for TC Rotor with FRB}

To obtain further insight into the dynamics of TC, it is useful to know the approximate normal modes and stability of the rotor system. Since the TC rotor is highly nonlinear due to the FRBs, in the strict sense it is hard to estimate the speed region of stability. To get approximate information on the vibration modes, the TC rotor with nonlinear FRBs should be linearized about an equilibrium position.

Table 3 Bearing damping coefficients

\begin{tabular}{|c|c|c|c|c|}
\hline Items & $C x x$ & $C x y$ & Cyx & Cyy \\
\hline Inner film $C_{i}(\mathrm{Ns} / \mathrm{mm})$ & 1.51 & $-6.97 \times 10^{-2}$ & $-6.97 \times 10^{-2}$ & 1.93 \\
\hline Outer film $C_{0}(\mathrm{Ns} / \mathrm{mm})$ & $5.18 \times 10^{-1}$ & $-3.64 \times 10^{-2}$ & $-3.64 \times 10^{-2}$ & $6.75 \times 10^{-1}$ \\
\hline
\end{tabular}


The new coefficients (inner and outer fluid-film dynamic coefficients for each bearing) were employed in the rotor model of Figure 3 to get the eigenvalues and their eigenvectors, and hence the stability characteristics of the system. The stability behavior of the rotor was discussed by run-up simulation, where the rotor speed linearly increased from $10000 \mathrm{r} / \mathrm{min}$ up to $220000 \mathrm{r} / \mathrm{min}$ with increment $6000 \mathrm{r} / \mathrm{min}$. The QR algorithm was utilized in the calculation of eigenvalues and eigenvectors, which had been well proven to be reliable and numerically stable. The imaginary parts of the eigenvalues were the system damped natural frequencies and they could be used to determine the damped critical speeds. The real parts of the eigenvalues were the system damping coefficients which could be used to determine the system stability. The logarithmic decrements were used to determine the system stability. A negative logarithmic decrement indicated that system was instability. The stability map of the TC rotor with linearized FRBs is shown in Figure 4. It also shows the first six logarithmic decrements of eigenvalues of the rotor system as a function of the rotational speed.

It can be seen from Figure 4 that there is only one mode of instability nearly at low speeds and two modes

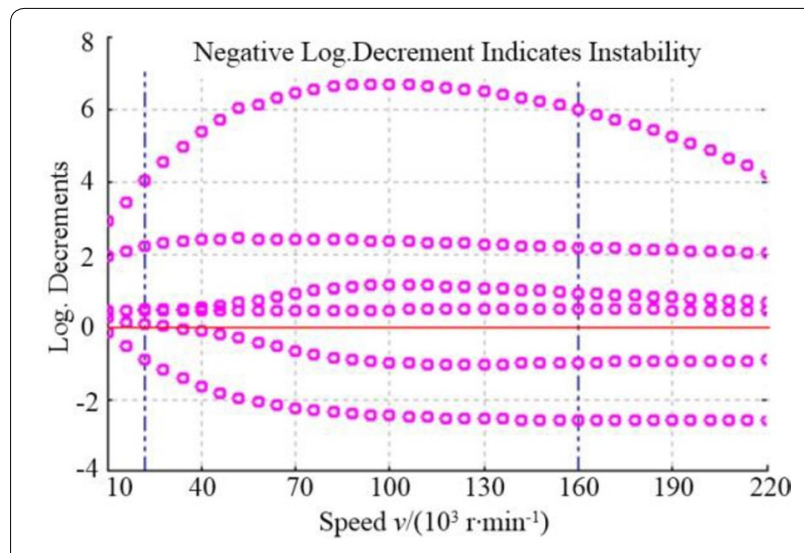

Figure 4 Stability map of TC rotor with linearized FRBs of instability at higher speeds. The threshold of instability is about $30000 \mathrm{r} / \mathrm{min}$. In fact, the threshold of instability is only defined in connection with a linearization of the perfectly balanced rotor around a stable equilibrium position and corresponding eigenvalue analysis. For the stability analysis with nonlinear bifurcation analyses of high-speed TC rotor with FRB, bifurcation from a stable equilibrium position (more generally unbalance vibrations around a stable equilibrium position) to the fully developed oil whirl/whip region with bearing eccentricities close to 1 may be rather sophisticated and depends on various system parameters, initial conditions, etc.

Figure 5 shows the instability modes with corresponding damped natural frequency $\omega_{d}$ as the rotational speed $N_{s}$. The damped natural frequency $\omega_{d}$ and corresponding mode shapes strongly depend on the rotor speed $N_{s}$. When the $N_{s}$ is $22000 \mathrm{r} / \mathrm{min}$, the $\omega_{d}$ is $13400 \mathrm{r} / \mathrm{min}$ and the forward rigid body conical mode with small elastic shaft bending is unstable. At speed $N_{s} 160000 \mathrm{r} / \mathrm{min}$, the forward conical mode ( $\omega_{d}$ is $42800 \mathrm{r} / \mathrm{min}$ ) is also unstable in addition to the approximately rigid body translational mode $\left(\omega_{d}\right.$ is $\left.61500 \mathrm{r} / \mathrm{min}\right)$. The second mode of instability with elastic shaft bending is obviously larger compared with conical mode.

\section{Simulations and Discussions}

The run-up simulation results concerning the effect of induced unbalance for an automotive high-speed TC rotor with FRBs are shown. Variations of the unbalance magnitude as well as distribution are considered in the performed simulations. The magnitude of the imposed unbalance $m e$ from $0.05 \mathrm{~g} \cdot \mathrm{mm}$ to $0.4 \mathrm{~g} \cdot \mathrm{mm}$ with an increasing ratio 2 (see Table 4) for both disks, and the value of unbalance magnitude was decided by the statistical data from the results of high-speed dynamic balancing. The frequency domain of time transient analysis was taken at speeds range from $10000 \mathrm{r} / \mathrm{min}$ to $220000 \mathrm{r} / \mathrm{min}$ based on the FEM with difference induced unbalance magnitudes on the nose wall of turbine and compressor

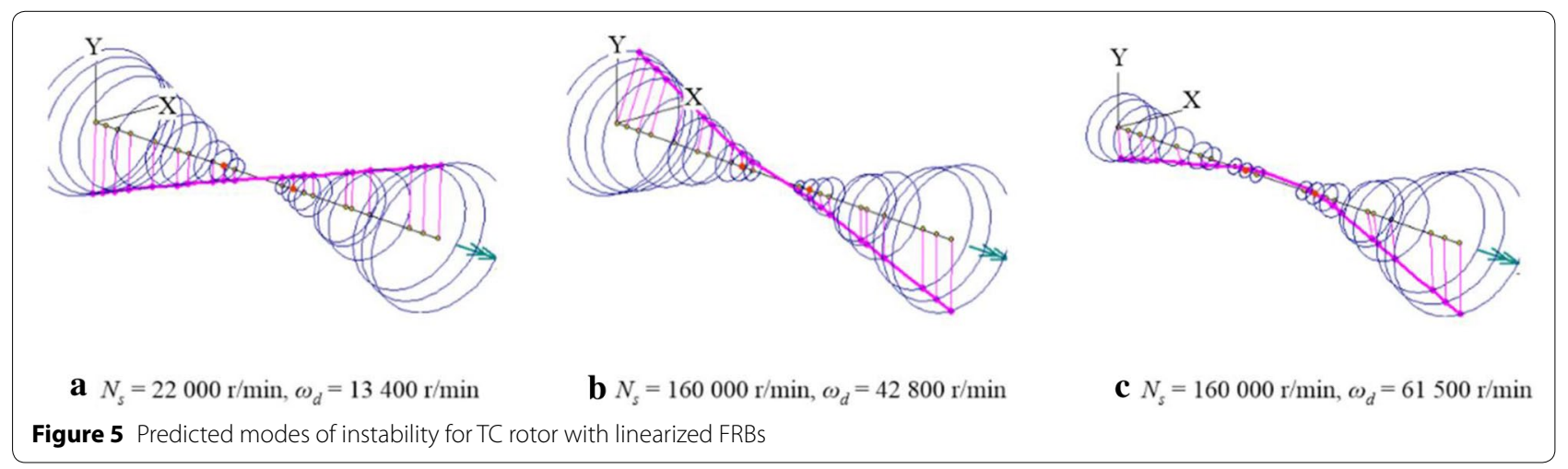


wheels (stns 4 and 18) to investigate the dynamic behavior of TC rotor system. The Newmark-beta method was chosen as integration method for the time transient analysis, which was well-known constant-average-acceleration method. Note that level 1 to 4 represent the induced unbalance magnitude on the stns 4 and 18 of the FEM from Figure 3, respectively.

\subsection{Induced Unbalance on Turbine Wheel}

The run-up simulation results for the horizontal displacement at stn 20 for four different levels of induced unbalance magnitude on the stn 4 are depicted in Figure 6. And the response spectra intensity plots also are presented in Figure 7, in which the following colour pattern corresponds to the ascending order of the response magnitude: yellow, light blue, dark blue, and red. The results of simulation are summarised as follows.

Table 4 Four different magnitudes of induced unbalance

\begin{tabular}{lllll}
\hline Level & $\mathbf{1}$ & $\mathbf{2}$ & $\mathbf{3}$ & $\mathbf{4}$ \\
\hline Unbalance $U(\mathrm{~g} \cdot \mathrm{mm})$ & 0.05 & 0.1 & 0.2 & 0.4 \\
\hline
\end{tabular}

(1) When unbalance is small with $0.05 \mathrm{~g} \cdot \mathrm{mm}$, there are four sub-synchronous frequency components: $0.5 \times, 0.12 \times, 0.3 \times$, and $0.37 \times$ from Figure $6(\mathrm{a})$ and Figure $7(\mathrm{a})$. Note that the whirl speed with sub $0.5 \times$ which mainly caused by inner oil film approximately equals to the half of rotor speed, and the predominated $0.12 \times$ response which mainly caused by outer oil film equals to the half of the ring speed. However, the whirl speed with sub $0.3 \times$ which mainly caused by inner and outer oil film approximately equals to the half of the whirl speed $0.5 \times$ and $0.12 \times$, and sub $0.37 \times$ which mainly caused by inner and outer oil film approximately equals to the half of the whirl speed $0.5 \times$ and $0.24 \times$.

(2) When unbalance magnitude increases to $0.1 \mathrm{~g} \cdot \mathrm{mm}$, the waterfall plot from Figure 6(b) just exist sub $0.5 \times, 0.12 \times$, and $0.37 \times$. The $1 \times$ grows but is very small at the low speed. The onset speed of the sub $0.12 \times$ is $94000 \mathrm{r} / \mathrm{min}$ for the unbalance $0.1 \mathrm{~g} \cdot \mathrm{mm}$ and $85000 \mathrm{r} / \mathrm{min}$ for the unbalance $0.05 \mathrm{~g} \cdot \mathrm{mm}$ from Figure 7(a) and (b), respectively. Then the predominated $0.12 \times$ response increases rapidly with the rotational speed and turns to a conical forward
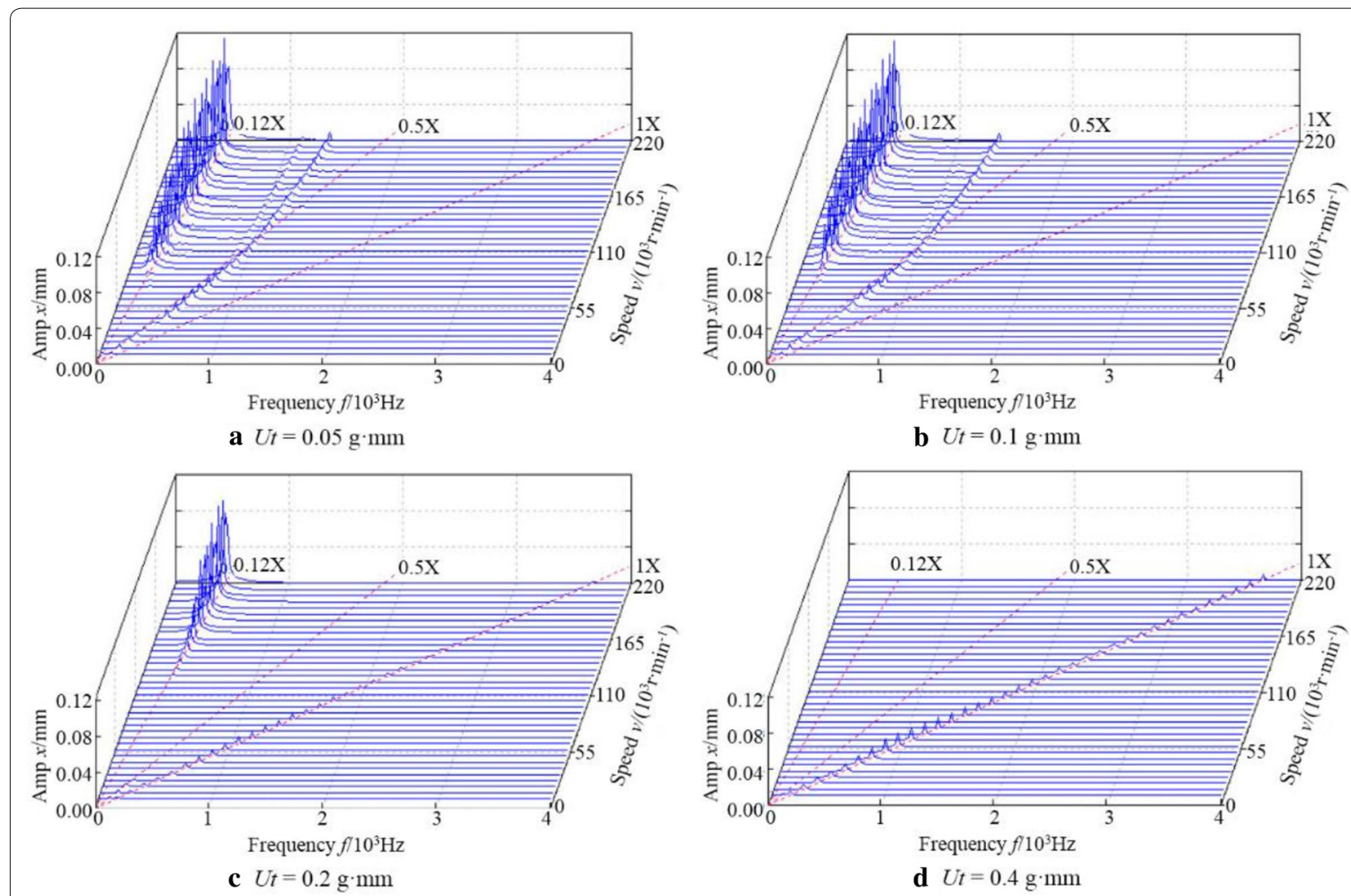

Figure 6 Waterfall plots of stn 20 for four difference unbalance amplitudes in TW nose of rotor 

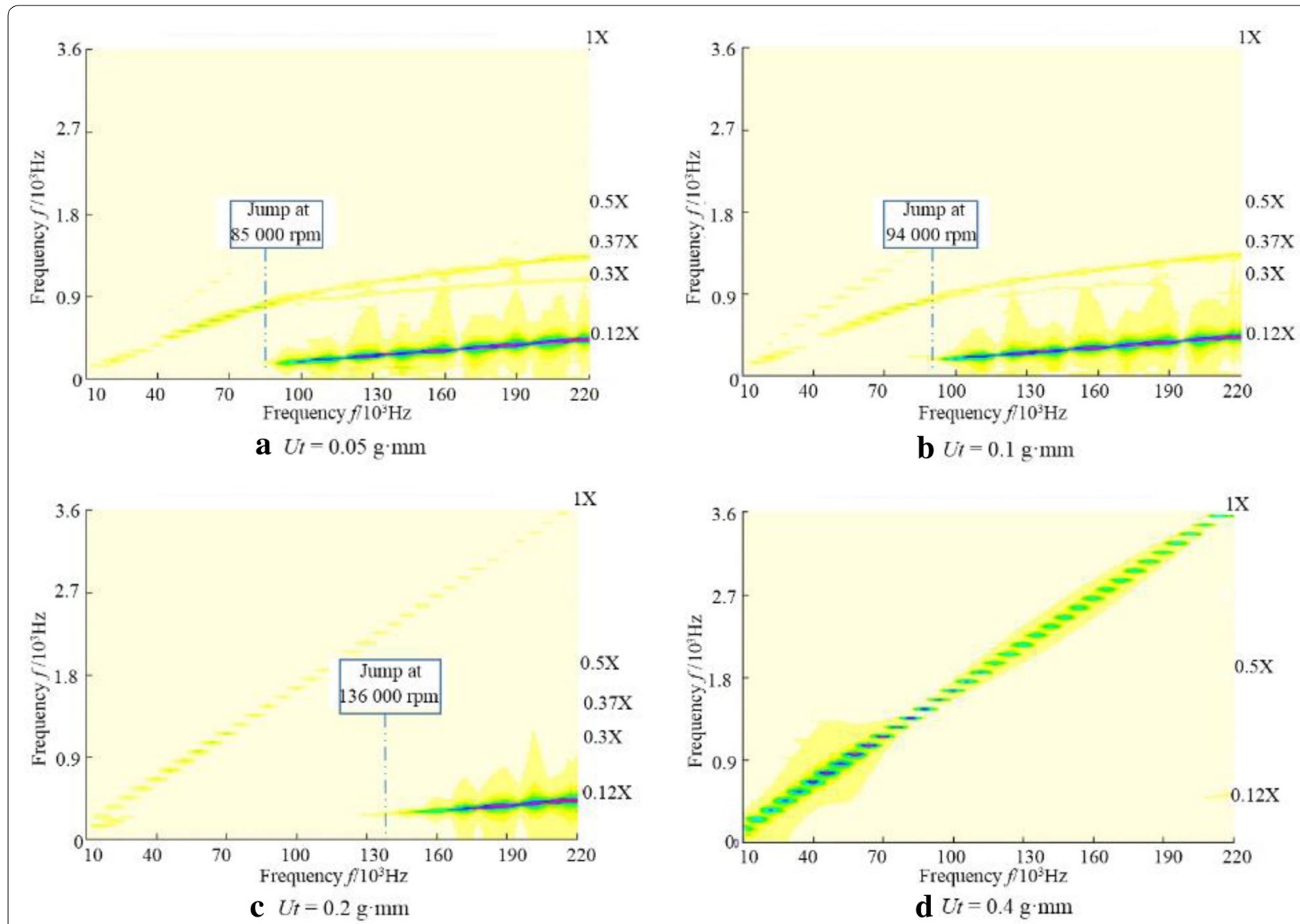

Figure 7 Response spectral intensity plots of stn 20 for four different unbalance amplitudes in TW nose of rotor

mode, due to the instability of outer films at both FRBs.

(3) When unbalance magnitude is not big, the TC rotor with FRBs system becomes unstable at the start of simulation, which is confirmed in Figure 6(a) and (b). This instability is originated from the instability of inner films with sub $0.5 \times$, and Figure 5(a) shows that a conical mode is excited. With increasing rotor speed, this instability can be pass through, and the system becomes unstable again which the dominant frequency component jumps from the sub $0.5 \times$ to $0.12 \times$. The presence of instability sub $0.5 \times$ is bifurcated as two new instability sub $0.3 \times$ and $0.37 \times$ which their influence is less than sub $0.12 \times$.

(4) Figure 6(c) and (d) show that higher unbalance magnitude values, e.g., $0.2 \mathrm{~g} \cdot \mathrm{mm}$ and $0.4 \mathrm{~g} \cdot \mathrm{mm}$, can suppress sub $0.5 \times$ at the very beginning of the runups compared with Figure 6(a) and (b). The onset speed of the sub $0.12 \times$ delays to approximately $136000 \mathrm{r} / \mathrm{min}$ for the induced unbalance $0.2 \mathrm{~g} \cdot \mathrm{mm}$ and disappears for the $0.4 \mathrm{~g} \cdot \mathrm{mm}$ from Figure 7 (c) and (d), respectively. Obviously, the rotor undergoes purely unbalance induced vibrations for the enough unbalance magnitude. Moreover, the total vibration value of TC rotor decreases with increasing of induced unbalance magnitude, and the subsynchronous frequency components can be suppressed.

\subsection{Induced Unbalance on Compressor Wheel}

Figures 8 and 9 show the results of the run-ups when the variable values of the induced unbalance magnitude are imposed on compressor wheel. In comparison with the induced unbalance magnitude on turbine wheel case described in Section 4.1, the conclusions of the obtained results are generally drawn as follows.

(1) Compared with the unbalance magnitude on turbine wheel, the synchronous $1 \times$ exists even in the condition with small unbalance $(0.05 \mathrm{~g} \cdot \mathrm{mm}$ 
and $0.1 \mathrm{~g} \cdot \mathrm{mm}$ ) and its amplitude is higher for the corresponding induced unbalance magnitude on compressor wheel. When the induced unbalance magnitude is the smallest, there are three sub-synchronous frequency components: $0.5 \times, 0.12 \times$, and $0.3 \times$ from Figure 8(a) and Figure 9(a). Meantime, the rotor motion is predominated by the sub $0.12 \times$.

(2) When unbalance magnitude increases to $0.1 \mathrm{~g} \cdot \mathrm{mm}$, the waterfall plot from Figure 8(b) just exists sub $0.5 \times$ and $0.12 \times$. The $0.5 \times$ just exists in the beginning of run-up but its amplitude is very small. The onset speed of the predominated $0.12 \times$ is about $88000 \mathrm{r} / \mathrm{min}$ for the unbalance $0.05 \mathrm{~g} \cdot \mathrm{mm}$ and $133000 \mathrm{r} / \mathrm{min}$ for the unbalance $0.1 \mathrm{~g} \cdot \mathrm{mm}$ from Figure $9(\mathrm{a})$ and (b), respectively. Then the sub $0.12 \times$ frequency vibration increases rapidly with the rotational speed and also turns to a conical forward mode, due to the instability of outer films at both FRBs.

(3) When unbalance magnitude is not big, the TC rotor with FRBs system becomes unstable at the start of run-up simulation, which is confirmed in Figure $8(\mathrm{a})$ and (b). This instability is also due to the sub $0.5 \times$, and Figure 5(a) shows is a conical mode. At $88000 \mathrm{r} / \mathrm{min}$, the dominant frequency component jumps from sub $0.5 \times$ to sub $0.12 \times$ from Figure 9 (a). It is very interesting that the presence of instability sub $0.5 \times$ is bifurcated as two new instability sub $0.12 \times$ and $0.3 \times$. Moreover the sub $0.12 \times$ is a conical mode shown in Figure 5(b), and the sub $0.3 \times$ is a bending cylindrical mode shown in Figure $5(\mathrm{c})$.

(4) Figure 8(c) and (d) show that higher unbalance magnitude on the compressor wheel, e.g., $0.2 \mathrm{~g} \cdot \mathrm{mm}$ and $0.4 \mathrm{~g} \cdot \mathrm{mm}$, obviously can suppress sub $0.5 \times$ at the beginning of the run-ups and becomes mainly the $1 \times$ caused by the large unbalance excitation. Moreover, the total vibration value of TC rotor increases with the increasing of induced unbalance magnitude because the rotor only predominates purely unbalance vibration mode.

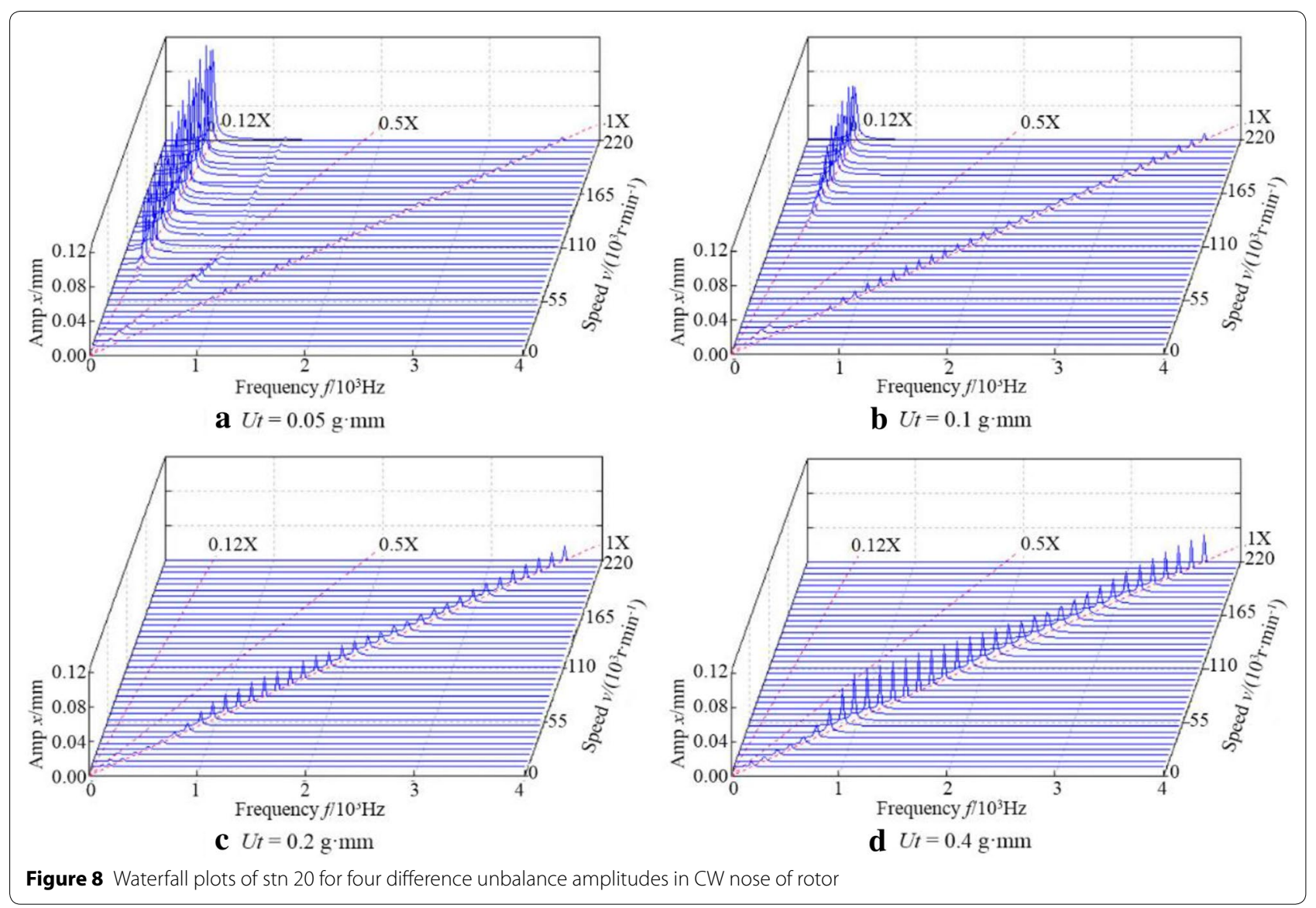



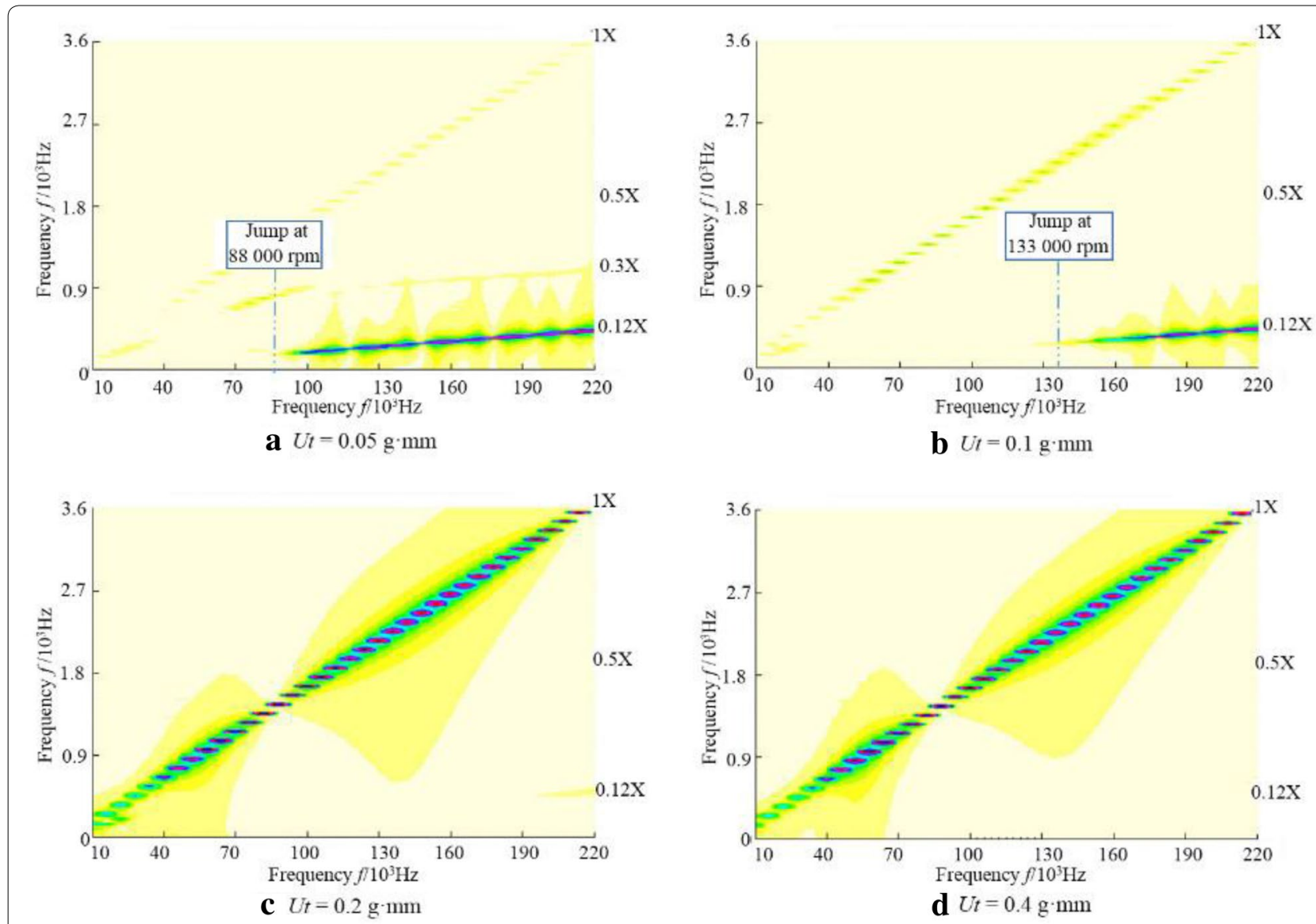

Figure 9 Response spectral intensity plots of stn 20 for four difference unbalance amplitudes in CW nose of rotor

\section{Conclusions}

(1) The waterfall and response spectral intensity plots are obvious different for the four different unbalance magnitudes. The onset speed of sub-synchronous frequency is not identical and the total vibration amplitude is different with the variable unbalance. Moreover, the sub $0.12 \times$ is the dominant frequency for the small induced unbalance magnitude. Large magnitude of low-frequency whirl due to the sub-synchronous frequency may lead to the instability of the high-speed TC rotor. The induced unbalance magnitude should be controlled in the running process for the high speed TC rotor with FRBs.

(2) The bifurcation and instability phenomena occur during the startup strongly depends on the induced unbalance magnitude. For the small unbalance magnitude, the rotor motion is predominated by the sub-synchronous components and the synchronous vibration is small. The bifurcation phenomena are nearly consistent for the induced unbal- ance on turbine wheel and compressor wheel, but the sub-synchronous responses caused by unbalance inducing the turbine wheel is stronger than the compressor wheel for the same level of unbalance magnitude. The main difference for the biggest unbalance magnitude $(0.4 \mathrm{~g} \cdot \mathrm{mm})$ is that all sub-synchronous frequencies disappear and just only exist synchronous response $1 \times$, which the high-speed TC rotor with FRBs performs the pure unbalance oscillations around the equilibrium position.

(3) The sub-synchronous frequencies can make the rotor unstable and cause large vibration for the TC rotor with small unbalance magnitude. The main reason for the excessive vibration is caused by the oil film asynchronous vibration. The oil whirl/whip can be suppressed for the high speed range of TC. Meantime, the sub-synchronous frequencies vibration can be suppressed by a suitable unbalance magnitude. However, as unbalance increases from $0.2 \mathrm{~g} \cdot \mathrm{mm}$ to $0.4 \mathrm{~g} \cdot \mathrm{mm}$, the rotor response amplitude increases too and becomes mainly the $1 \times$ caused by the large unbalance excitation. Therefore, 
a suitable unbalance magnitude of turbine wheel and compressor wheel is proposed. To control the induced unbalance magnitude is an effective way to achieve the small vibration and stable operation for the high-speed TC rotor with FRBs.

\section{Authors' Contributions}

G-FB and YH was in charge of the whole trial; G-FB and X-JL wrote the manuscript; G-FB and S-PG assisted with sampling and laboratory analyses. All authors read and approved the final manuscript.

\section{Author Details}

${ }^{1}$ Health Maintenance for Mechanical Equipment Key Lab of Hunan Province, Hunan University of Science and Technology, Xiangtan 411201, China. 2 Department of Mechanical and Aerospace Engineering, The University of Alabama in Huntsville, Huntsville, AL 35899, USA

\section{Authors' Information}

Guang-Fu Bin, born in 1981, is currently an associate professor at Health Maintenance for Mechanical Equipment Key Lab of Hunan Province, Hunan University of Science and Technology, China. He acted as an academic visitor in University of Ottawa from 2008 to 2009. He received his PhD degree from Beijing University of Chemical Technology, China, in 2013. His research interests include rotating machinery dynamics and modal analysis, shafting dynamic balance, mechanical dynamic testing.

Yuan Huang, born in 1993, is currently a master candidate at Health Maintenance for Mechanical Equipment Key Lab of Hunan Province, Hunan University of Science and Technology, China. His research interests include rotordynamics, automotive turbochargers and oil film bearing.

Shuai-Ping Guo, born in 1987, is currently a lecturer at Health Maintenance for Mechanical Equipment Key Lab of Hunan Province, Hunan University of Science and Technology, China. He received his PhD degree from Hunan University, China, in 2015. His research interests include mechanical dynamics, buckling analysis, heat conduction, boundary element method.

Xue-Jun Li, born in 1969, is currently a professor at Health Maintenance for Mechanical Equipment Key Lab of Hunan Province, Hunan University of Science and Technology, China. He received his PhD degree from Central South University, China, in 2003. He received post-doctor degree from Tsinghua University, China, in 2009. His main research interests include mechanical dynamics and fault diagnosis, signal analysis and processing.

Gang Wang, born in 1971, is currently an associate professor at Department of Mechanical and Aerospace Engineering, the University of Alabama in Huntsville, Huntsville, USA. He received his PhD degree from University of Maryland at College Park, USA, in 2001. His main research interests include mechanical dynamics and fault diagnosis, signal analysis and processing.

\section{Competing Interests}

The authors declare that they have no competing interests.

\section{Funding}

Supported by National Natural Science Foundation of China (Grant Nos. $51575176,11672106,51775030,51875196)$, and Youth Innovative Talents of Hunan Province of China (Grant No. 2015RS4043)

\section{Publisher's Note}

Springer Nature remains neutral with regard to jurisdictional claims in published maps and institutional affiliations.

Received: 14 June 2016 Accepted: 26 September 2018

Published online: 16 October 2018

\section{References}

[1] C J Kim, Y J Kang, B H Lee, et al. Determination of optimal position for both support bearing and unbalance mass of balance shaft. Mechanism and Machine Theory, 2012, 50(50): 150-158.

[2] L S Andres, J C Rivadeneira, M Chinta, et al. Nonlinear rotordynamics of automotive turbochargers: predictions and comparisons to test data. ASME Journal of Engineering for Gas Turbines and Power, 2005, 129(2): 488-493.

[3] G C Ying, G Meng, J P Jing. Turbocharger rotor dynamics with foundation excitation. Archive of Applied Mechanics, 2009, 79(4): 287-299.

[4] S Rohde, H Ezzat. Analysis of dynamically loaded floating-ring bearings for automotive applications. Journal of Lubrication Technology, 1980 102(3): 271-277.

[5] N S Hung. Rotordynamics of automotive turbochargers. Berlin: Springer Berlin Heidelberg, 2012

[6] R G Kirk, A Alsaeed, E J Gunter. Stability analysis of a high-speed automotive turbocharger. Proceedings of the ASTLE / ASME International Joint Tribology Conference, San Antonio, TX, United States, October 23-25, 2006.

[7] Q H Li, W M Wang, PY Qi, et al. Stability analysis and identification method for rotor-bearings system. Journal of Mechanical Engineering, 2014, 50(7): 54-59. (in Chinese)

[8] A Boyaci, W Seemann, C Proppe. Stability analysis of rotors supported by floating ring bearings. The 8th IFTOMM International Conference on Rotor Dynamics, Seoul, Korea, 2010: 286-295.

[9] B Schweizer. Dynamics and stability of turbocharger rotors. Archive of Applied Mechanics, 2010, 80(9): 1017-1043.

[10] B Schweizer. Total instability of turbocharger rotors-physical explanation of the dynamic failure of rotors with full - floating ring bearings. Journal of Sound and Vibration, 2009, 328(1-2): 156-190.

[11] L Tian, W J Wang, Z J Peng. Effects of bearing outer clearance on the dynamic behaviors of the full floating ring bearing supported turbocharger rotor. Mechanical Systems and Signal Processing, 2012, 31 (8): 155-175.

[12] L M Zhai, Y Y Luo, Z W Wang, et al. Failure analysis and optimization of the rotor system in a diesel turbocharger for rotor speed-up test. Advances in Mechanical Engineering, 2014, 6(4): 476023-1-8.

[13] E J Gunter, W J Chen. Dynamic analysis of a turbocharger in floating bushing bearings. Proceedings of the 3rd International Symposium on Stability Control of Rotating Machinery, Cleveland, Ohio, USA, September 19-23, 2005.

[14] A A Alsaeed. A study of methods for improving the dynamic stability of high-speed turbochargers. Virginia: Virginia Polytechnic Institute and State University, 2010.

[15] Sterling, A John. Influence of induced unbalance on subsynchronous vibrations of an automotive turbocharger. Blacksburg: Virginia Polytechnic Institute and State University, 2009.

[16] R G Kirk, A A Alsaeed. Induced unbalance as a method for improving the dynamic stability of high-speed turbochargers. International Journal of Rotating Machinery, 2011, 2011: 1-9.

[17] L Tian, W J Wang, Z J Peng. Nonlinear effects of unbalance in the rotorfloating ring bearing system of turbochargers. Mechanical Systems and Signal Processing, 2013, 34(1-2): 298-320.

[18] J F Yao, J J Gao, W M Wang. Multi-frequency rotor vibration suppressing through self-optimizing control of electromagnetic force. Journal of Vibration and Control, 2015, 21:1-15.

[19] G B Wang, W H Deng, XY Du, et al. The absolute deviation rank diagnostic approach to gear tooth composite fault. Shock and Vibration, 2017, 2017(17): 1-10.

[20] H Ma, F L Yin, XY Tai, et al. Vibration response analysis caused by rubbing between rotating blade and casing. Journal of Mechanical Science and Technology, 2016, 30(5): 1983-1995.

[21] W J Chen. Rotordynamics and bearing design of turbochargers. Mechanical Systems and Signal Processing, 2012, 29(5): 77-89.

[22] W J Chen, E J Gunter. Introduction to dynamics of rotor-bearing systems. Victoria: Trafford Publishing, 2005. 